\section{Joint channel prediction aided differentially encoded TTCM and BICMID assisted eigen-beamforming}

\author{
W. Liu, S.X. Ng, L.L. Yang and L. Hanzo
}

Eigen-beamforming is capable of providing attractive performance gains in the context of multiple-input multiple-output systems, provided accurate channel state information (CSI) is available. However, when a realistic pilot-based channel predictor is used for acquiring the CSI, a significant performance degradation may be imposed by the phase-ambiguity inherent in the estimated eigenvectors. Sophisticated differentially encoded coded modulation (CM) schemes are amalgamated with the eigen-beamformer, when employing a minimum mean square error based pilot-assisted channe predictor for avoiding the performance degradation imposed by outdated channel estimation. It is shown that the amalgamated differentially encoded $\mathrm{CM}$ aided eigen-beamformer attained coding gain of about $6.5 \mathrm{~dB}$, when communicating over correlated Rayleigh fading channels

Introduction: Wireless systems employing multiple transmitters and receivers are capable of providing high data rates by exploiting the high capacity potential of multiple-input multiple-output (MIMO) channels [1]. Within the broad family of MIMO systems, space-time coding schemes [2] are capable of attaining attractive spatial diversity gains without requiring any channel state information (CSI) at the transmitter. By contrast, transmit eigen-beamforming [3] requires accurate CSI at the transmitter for guaranteeing low-SNR operation.

CSI can be estimated at the receiver and fed back to the transmitter, but outdated CSI estimation may seriously degrade the attainable performance [4]. Alternatively, instead of awaiting the outdated channel estimates, CSI prediction may be used for acquiring the required CSI [4]. Therefore, in this Letter we employ the latter approach, using a lowcomplexity minimum mean square error (MMSE) based pilot-symbol aided MIMO channel predictor [5]. However, the CSI provided by a practical channel predictor used by the eigen-beamformer is prone to the phase-ambiguity inherent in the predicted eigen-vectors [6], which will be counteracted by differentially encoded modulation [7].

Against this background, the novel contribution of this Letter is that for the sake of achieving a high bandwidth efficiency, eigenbeamforming is amalgamated with differentially encoded turbo trellis coded modulation (TTCM) [7] and iteratively decoded bit-interleaved coded modulation (BICMID) [7], demonstrating that the proposed scheme using realistic channel prediction and 8PSK BICMID is capable of outperforming the idealistic perfect CSI estimation based 4PSK benchmarker for SNRs in excess of $10 \mathrm{~dB}$ without any bandwidth expansion. The proposed differentially encoded schemes outperform their coherently detected counterparts, which suffer from effects of phase ambiguity.

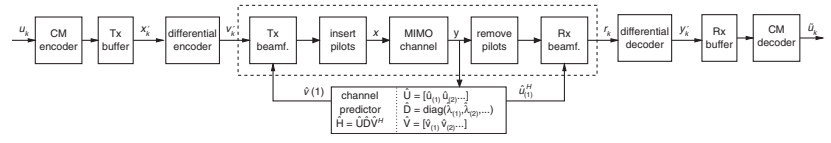

Fig. 1 Block diagram of channel prediction aided differential CM assisted eigen-beamforming system

System overview: Consider a system employing $M_{T}$ transmit and $M_{R}$ receive antennas for communicating over flat Rayleigh fading channels. The block diagram of the channel prediction aided differentially encoded CM assisted eigen-beamforming scheme is shown in Fig. 1, where $($.$) denotes the predicted value of ($.$) , while (\sim)$ denotes the detected value of (.). Transmit and receive buffers are used for storing an encoded frame, which is partitioned into shorter subframes. $M_{T}$ number of pilot symbols are attached to each subframe at its beginning [5], as seen in Fig. 1. A shorter subframe length is expected to increase the accuracy of the channel predictor, but naturally, it also imposes a higher pilot symbol overhead. The $M_{R}$-dimensional vector of received symbols $\mathbf{y}$ can be expressed as $\mathbf{y}=\mathbf{H x}+\mathbf{n}$, where $\mathbf{x}$ is the $M_{T}$-dimensional vector of transmitted symbols and $\mathbf{H}$ is an $\left(M_{R} \times M_{T}\right)$-dimensional complex-valued channel matrix, while $\mathbf{n}$ is the $M_{R}$-dimensional AWGN vector. If the rank of $\mathbf{H}$ is assumed to be $q$, the singular value decomposition (SVD) of $\mathbf{H}$ results in [1]
$\mathbf{H}=\mathbf{U D V} \mathbf{V}^{H}$, where $\mathbf{D}=\operatorname{diag}\left(\lambda_{(1)}, \ldots, \lambda_{(q)}\right)$, while the $\{\lambda\}$ s are the singular values of $\mathbf{H}$. Furthermore, $\mathbf{U}$ and $\mathbf{V}$ are $\left(M_{r} \times q\right)$ and $\left(M_{t} \times q\right)$ unitary matrices, respectively.

A sequence of information symbols $\left\{u_{k}\right\}$, where the subscript $k$ denotes the time index, is first TTCM- or BICMID-encoded to generate the sequence $\left\{x_{k}^{\prime}\right\}$, before entering the differential encoder, where the sequence $\left\{v_{k}\right\}$ is produced. At the transmit beamforming block of Fig. $1, v_{k}$ is multiplied by the beamforming weight vector $v_{(1) k}$ to produce $\mathbf{x}_{k}$, where $\mathbf{v}_{(1) k}$ is the first column vector of the unitary matrix $\mathbf{V}$ computed using the SVD. At the receiver, receive beamforming is carried out with the aid of the beamforming weight vector of $\mathbf{u}_{(1) k}^{H}$, which is the conjugate transpose of the first column vector of the unitary matrix $\mathbf{U}$. Differential decoding is then carried out, followed by TTCM or BICMID decoding, as seen in Fig. 1.

Simulation results: Let us consider transmissions over correlated Rayleigh fading channels having a normalised Doppler frequency of $10^{-3}$ and a subframe length of $L_{s}=100$ TTCM or BICMID-encoded symbols as well as a frame length of $L_{f}=1000 \mathrm{CM}$-encoded symbols. The predictor order was fixed to $p=10$. Coherently detected CM or differential-encoded CM (D-CM) schemes were employed. The number of transmit antennas was fixed to $M_{T}=2$ and the number of receive antennas was also $M_{R}=2$. The pilot overhead was given by $M_{T} /\left(M_{T}+L_{s}\right)$, which is $1.96 \%$, when using $M_{T}=2$ and $L_{s}=100$.

For fair comparison, both of the $\mathrm{CM}$ schemes employed were configured to have a similar decoding complexity quantified in terms of the total number of trellis decoding states. Since TTCM schemes invoke two TCM component codes, a TTCM code employing $t$ iterations and using an $S$-state component code exhibits a complexity proportional to 2.t.S or $t .2^{v+1}$ states. As for BICMID schemes, only one decoder is used, but the demodulator is invoked in each decoding iteration. However, the complexity of the demodulator is assumed to be insignificant compared to that of the trellis decoder. Hence, a BICMID code employing $t$ iterations and using an $S$-state code exhibits a complexity proportional to $t . S$ or $t .2^{M}$. For these reasons, we opted for $S=8$ and $t=8$ for the BICMID scheme. The code polynomials used for the CM schemes can be found on pages 792 and 798 in [7].

Fig. 2 shows the bit error ratio (BER) against signal-to-noise ratio (SNR) per bit, namely $E_{b} / N_{0}$, performance of the 4PSK and CM-8PSK assisted eigen-beamforming schemes, when employing either perfect or predicted CSI without differential encoding. Hence, as seen from Fig. 2, the coherently detected scheme suffers from a significant performance degradation, when the CSI is imperfect. For example, at BER $=10^{-5}$ an approximately $8.5 \mathrm{~dB}$ and $6.2 \mathrm{~dB}$ performance loss is incurred, when employing the predicted CSI compared to the scheme benefiting from perfect CSI for the 4PSK and TTCM-8PSK assisted schemes, respectively. This is due to the phase-ambiguity problem.

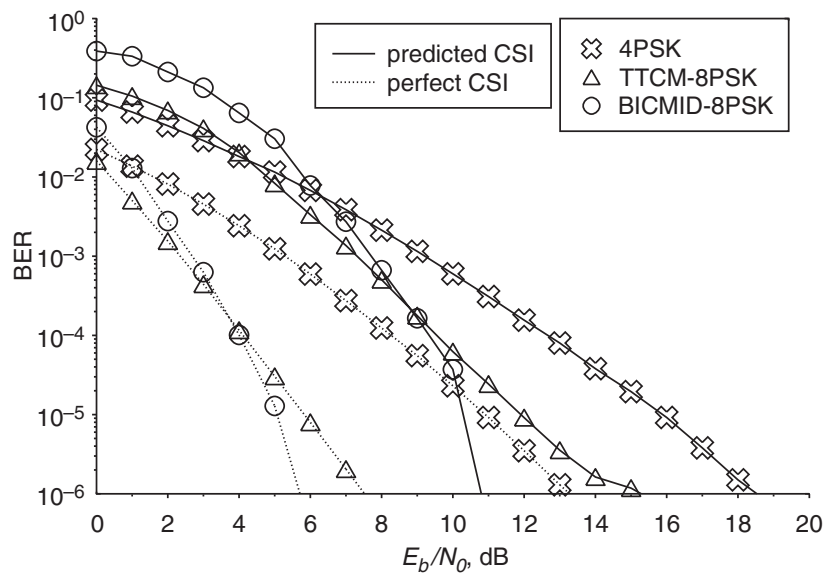

Fig. 2 BER against $E_{b} / N_{0}$ performance of $4 P S K\left(L_{f}=L_{s}=100\right)$ and CM-8PSK $\left(L_{f}=1000, L_{s}=100\right)$ beamforming schemes, when communicating over correlated Rayleigh fading channels having normalised Doppler frequency of $10^{-3}$ (pilot overhead 1.96\%)

Fig. 3 shows the performance of the 4PSK and CM-8PSK assisted eigen-beamforming schemes with and without differential coding. As seen from Fig. 3, when the CSI is imperfect, the D-CM-8PSK scheme performs better than the CM-8PSK arrangement. For example, at 
$\mathrm{BER}=10^{-5}$ the D-BICMID-8PSK scheme using realistic CSI prediction performs about $1 \mathrm{~dB}$ better than its coherently detected nondifferential counterparts owing to the deleterious effects of phase ambiguity. Furthermore, the uncoded D-4PSK scheme has a similar performance to that of the uncoded 4PSK scheme, as evidenced in Fig. 3, when the CSI was imperfect. These findings underline the importance of using error correction schemes in practical eigenbeamforming schemes. More specifically, observe in Fig. 3 that at BER of $10^{-5}$, the D-BICMID-8PSK scheme attained a coding gain of about $6.5 \mathrm{~dB}$ over both the 4PSK and D-4PSK schemes, when using realistic predicted CSI. Note that the CM schemes employed were suboptimum, since they were originally designed for coherently detected non-differential modulation, nonetheless their amalgamation with differential coding yielded attractive performance gains in the context of realistically predicted CSI.

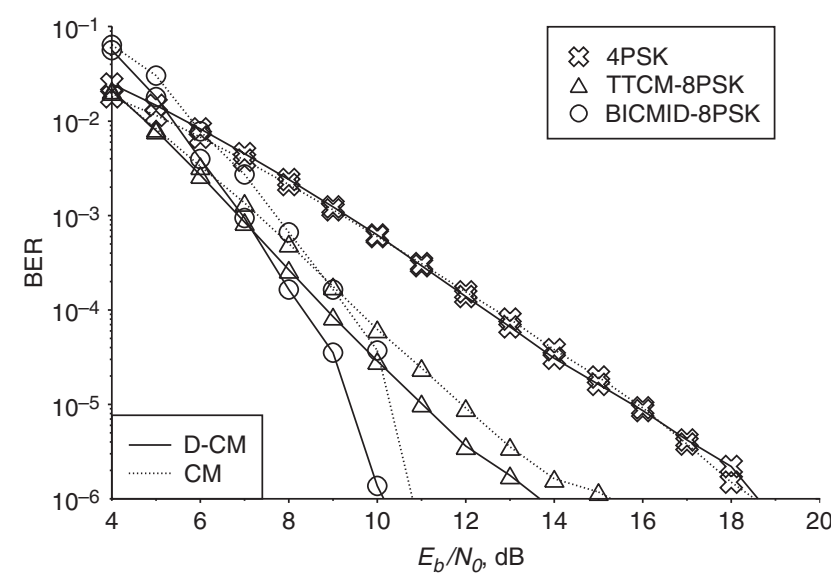

Fig. 3 BER against $E_{b} / N_{0}$ performance of $4 P S K\left(L_{f}=L_{s}=100\right)$ and CM-8PSK $\left(L_{f}=1000, L_{s}=100\right)$ beamforming schemes with and without differential coding, when communicating over correlated Rayleigh fading channels having normalised Doppler frequency of $10^{-3}$ with aid of MMSE channel predictor (Pilot overhead is 1.96\%).
Conclusion: We propose channel prediction aided differentially encoded TTCM and BICMID modulation amalgamated with eigenbeamforming, when communicating over correlated Rayleigh fading channels, which resulted in a $6.5 \mathrm{~dB} E_{b} / N_{0}$ gain.

(C) The Institution of Engineering and Technology 2007 19 October 2006

Electronics Letters online no: 20073252

doi: 10.1049/el:20073252

W. Liu, S.X. Ng, L.L. Yang and L. Hanzo (School of ECS, University of Southampton, SO17 1BJ, United Kingdom)

E-mail: 1h@ecs.soton.ac.uk

\section{References}

1 Telatar, E.: 'Capacity of multi-antenna Gaussian channels', Eur. Trans. Telecommun., 1999, 10, pp. 585-595

2 Alamouti, S.M.: 'A simple transmitter diversity scheme for wireless communications', IEEE J. Sel. Areas Commun., 1998, 16, pp. 1451-1458

3 Abdel-Samad, A., Davidson, T.N., and Gershman, A.B.: 'Robust transmit eigen beamforming based on imperfect channel state information', IEEE Trans. Signal Process., 2006, 54, pp. 1596-1609

4 Choi, L., and Murch, R.D.: 'Transmit-preprocessing techniques with simplified receivers for the downlink of MISO TDD-CDMA systems', IEEE Trans. Wirel. Commun., 2004, 35, pp. 285-295

5 Luo, Z., Gao, H., Liu, Y., and Gao, J.: 'Robust pilot-symbol-aided MIMO channel estimation and prediction'. IEEE Global Telecommunications Conf., Dallas, TX, USA, November-December 2004, pp. 3646-3650

6 Dahl, T., Christophersen, N., and Gesbert, D.: 'Blind MIMO eigenmode transmission based on the algebraic power method', IEEE Trans. Signal Process., 2004, 52, pp. 2424-2431

7 Hanzo, L., Ng, S.X., Webb, W., and Keller, T.: 'Quadrature amplitude modulation: from basics to adaptive trellis-coded, turbo-equalised and space-time coded OFDM, CDMA and MC-CDMA systems' (John Wiley and Sons, New York, 2004, 2nd edn.) 